\title{
Emotions, Arousal, and Frontal Alpha Rhythm Asymmetry During Beethoven's 5th Symphony
}

\author{
Christian Mikutta $\cdot$ Andreas Altorfer $\cdot$ \\ Werner Strik · Thomas Koenig
}

Received: 10 October 2011/Accepted: 6 April 2012/Published online: 26 April 2012

(C) Springer Science+Business Media, LLC 2012

\begin{abstract}
Music is capable of inducing emotional arousal. While previous studies used brief musical excerpts to induce one specific emotion, the current study aimed to identify the physiological correlates of continuous changes in subjective emotional states while listening to a complete music piece. A total of 19 participants listened to the first movement of Ludwig van Beethoven's 5th symphony (duration: $7.4 \mathrm{~min}$ ), during which a continuous 76-channel EEG was recorded. In a second session, the subjects evaluated their emotional arousal during the listening. A fast fourier transform was performed and covariance maps of spectral power were computed in association with the subjective arousal ratings. Subjective arousal ratings had good inter-individual correlations. Covariance maps showed a right-frontal suppression of lower alpha-band activity during high arousal. The results indicate that music is a powerful arousal-modulating stimulus. The temporal dynamics of the piece are well suited for sequential analysis, and could be necessary in helping unfold the full emotional power of music.
\end{abstract}

Keywords Arousal - Emotion $\cdot$ Music $\cdot$ EEG $\cdot$ Frontal alpha-asymmetry

C. Mikutta $(\bowtie) \cdot$ W. Strik

University Hospital of Psychiatry, University of Bern,

Bolligenstrasse 111, 3000 Bern 60, Switzerland

e-mail: christian.mikutta@spk.unibe.ch

A. Altorfer · T. Koenig

University Hospital of Psychiatry, Department of Psychiatric

Neurophysiology, University of Bern, Bolligenstrasse 111,

3000 Bern 60, Switzerland

\section{Introduction}

Music is a powerful inducer of emotions (Meyer 1956; Blood et al. 1999; Blood and Zatorre 2001; Koelsch et al. 2008; Juslin and Vastfjall 2008; Wu et al. 2010), and humans appear to listen to music specifically because of this (Panksepp and Bernatzky 2002; Menon and Levitin 2005). Furthermore, and in contrast to other methods of inducing emotions, music is capable of reliably evoking emotions with both positive and negative valence and varying arousal in nearly everybody (Koelsch 2005). It is thus particularly well suited for studying emotion, both in a healthy population and in patients who suffer from emotional dysregulation (Moradipanah et al. 2009). Further knowledge may also improve and help develop various therapeutic techniques (Madson and Silverman 2010; Zare et al. 2010).

Emotional states change rapidly during listening; emotions in music are therefore often assessed with dimensional models (Russel 1980; Zentner et al. 2008), with typical dimensions being valence and arousal level (Gable and Harmon-Jones 2009). Valence was used for evaluating whether a person liked or disliked certain musical pieces or excerpts (Koelsch 2005) and appears to be related to the presence of consonant or dissonant chords (Dellacherie et al. 2010). In contrast, arousal level seems to be independent of consonance or dissonance but strongly connected with loudness and musical expectation (Huron 2006).

In fact, specific harmonic or rhythmic sequences lead to determined expectations; for example, for a piece in $\mathrm{C}$ major, we expect that the $\mathrm{C}$ major tonic chord will be in its root position at closure of the piece. Such expectations may be explained, inter alia, due to statistical learning (Huron 2001). Music theorists have long claimed that violation of 
an expected chord usually increases emotional arousal, whereas the realization of that expectation lowers emotional arousal (Meyer 1956; Blood et al. 1999; Blood and Zatorre 2001; Patel 2003; Koelsch et al. 2008). Krumhansl (1995) showed, using a subjective rating for an ongoing melody, that expectation responses are independent of preexisting knowledge of the piece (intra-opus knowledge) or knowledge of the style (extra-opus knowledge).

On the other hand, arousal has been related to psychoacoustic parameters such as tempo (Frego 1999), dynamics (Ilie and Thompson 2006; Schubert 2004) or tessitura (Frego 1999). Thus, in the case of music, the induction of emotions may be due to an interaction of bottom-up (loudness, tempo, dynamics, etc.) and topdown expectations (Ochsner et al. 2009). Note however also that bottom-up and top-down processes may overlap and interact in ways that make a clear dichotomization difficult (Sarter et al. 2001).

Physiologically, it has been suggested that arousal is predominantly processed in the right hemisphere (Meadows and Kaplan 1994; Nitschke et al. 1999). A model proposed by (Craig 2005) suggested that this lateralization is due to an asymmetrical afference from the autonomic nervous system, where the right hemisphere receives preferentially sympathetic input that is associated with arousal, orienting responses and negative affect, whereas the left hemisphere receives mostly parasympathetic input that occurs predominantly during states of low arousal and positive affect. Evidence for such an asymmetry has long been found by EEG: In normally awake subjects, arousal, attention and orienting responses classically induce a suppression of alpha band activity (Krause 2006; Klimesch 1999; Jann et al. 2009). Accordingly, stimuli that are associated with a peripheral sympathetic response have been shown to induce a right lateralized suppression of frontal alpha band activity (Zhang et al. 2011; Allen et al. 2004; Davidson 2004).

In the music domain, Flores Guiterrez found also a lateralized frontal alpha suppression during listening of unpleasant and unpredictable music. The same group was also able to show that those activations are compatible with an activation of limbic sources (Flores-Gutierrez et al. 2007). Those findings are compatible with several other studies in which emotions were elicited through music. In those studies activation of amygdala, hippocampus, and nucleus accumbens as parts of the limbic system and further activation of paralimbic regions such as the orbitofrontal cortex, parahippocampal gyrus, cingulate gyrus, and temporal poles were found (Blood and Zatorre 2001; Koelsch et al. 2006, 2008; Eldar et al. 2007). Koelsch et al. (2006) found increased BOLD fMRI signals in the amygdala, hippocampus, parahippocampal gyrus, and temporal poles during unpleasant music, and that these emotion-related responses were independent of personal preferences. In another study, Koelsch et al. (2008) also showed that there was increased BOLD activation of the amygdala due to unexpected chords. Steinbeis et al. (2006) confirmed that such unexpected chords elicited an emotional response with increased electrodermal activity, which is compatible with states dominated by sympathetic activity. An alternative view on EEG correlates of emotional content is the approach-withdrawal model (Davidson 1995; Sammler et al. 2007). According to this model, frontal alpha assymetries are associated mostly with valence, whereas arousal is represented manly in right parietal systems (Heller et al. 1997). In this context, there is evidence for increased right parietal theta power during high arousal (Aftanas et al. 2002). This study used aversive stimuli from the International Affective Picture System (IASP) to evoke arousal, which is quite different to musical stimuli.

In summary, it is clear that emotion circuits, such as the limbic and paralimbic systems and the prefrontal cortex, are important for music-induced emotions and interact with cortically generated EEG rhythms.

Previous studies on music elicited emotions employed prototypical excerpts of music to elicit specific emotions (Tsang et al. 2001). However, this static view does not take advantage of the fact that much of music's excellent capacity for inducing emotions results from the sophisticated dynamics of emotional states that are elicited throughout a single piece of music. This constant flux of different musical and emotional states may prevent habituation and account for music's capacity to capture a subject's attention for any length of time, repeatedly (Huron 2006). This reliability is a valuable stimulus feature for the study of emotion.

Our aim was to identify using EEG the physiological correlates of the continuous changes in arousal during the listening of an entire and complex piece of music. This approach exploits the high time resolution of EEG (Muller et al. 1999; Tsuno et al. 2004) and takes arousal into account as a dimensional, and continuously changing rather than static and dimensional feature, which is probably a more naturalistic view. Furthermore, a focus on arousal is well justified both by a music-theoretical point of view as well as a by the knowledge about the physiology of processes activated during music listening.

In the present study, subjective arousal was examined during the entire first movement of Ludwig van Beethoven's 5th symphony (this piece of music was chosen because of its familiarity, thus minimizing individual differences in novelty). Previous findings showed that arousal was independent of consonant or dissonant chords (Dellacherie et al. 2010); therefore, we hypothesized that the subjective continuous arousal ratings would have neurophysiological correlates independent of the individual's 
like or dislike of the symphony. Specifically, we expected that that right frontal alpha power will decline during high music induced arousal (Heller et al. 1997; Allen et al. 2004; Davidson 2004). Further we expected higher theta power in bilateral parietal regions during states of arousal (Suetsugi et al. 2000; Aftanas and Golocheikine 2001). We further hypothesized that arousal ratings would be influenced by dynamic changes (loudness) in music, but that it would be possible to extract loudness independent EEG signatures of arousal. Furthermore, we tried to explore the influence of individual training on subjective arousal.

\section{Materials and Methods}

\section{Participants}

19 participants took part in the present study, selected from in-house staff and medical students. Mean age was 24 years (range 17-33). The inclusion criterion was current training in a musical instrument. Exclusion criteria were presence of central neurological disease, amblyacousia, psychiatric disorders, or psychotropic medication. All participants were right-handed. Amblyacousia was excluded by testing the auditory threshold of $5-10 \mathrm{~dB}$ at 2,000 Hz. Tests were performed using a Diatec screening audiometer AS-608. All participants played a musical instrument. 12 participants played piano, five played string instruments, one played pan-flute, and one played guitar. 14 played for more than 5 years. They all received music lessons as hobby-musicians in different styles (classical, jazz, modern music).

Participants practiced an average of $4.3 \mathrm{~h}$ per week (range $0.5-10 \mathrm{~h}$ ). Only one participant took part in a concert competition and intends to become a professional musician. All participants identified the music correctly as Ludwig van Beethoven's 5th symphony, but effects of the depth of knowledge of the music can of course not be excluded. The mean rating of like or dislike on a 10-point Likert-scale was 5.8 (max. 9, min. 3).

The study was approved by Canton's ethics committee and has therefore been performed in accordance with the ethical standards laid down in the 1964 Declaration of Helsinki and its later amendments. All subjects gave their informed consent prior to their inclusion in the study.

\section{Stimuli}

In the present study, the first movement of Ludwig van Beethoven's 5th symphony (duration: $442 \mathrm{~s}$ ) was used as the musical stimulus. Due to its extraordinary dynamics, the 1987 recording of the Vienna Philharmonic Orchestra conducted by Carlos Kleiber was selected. Music was presented via in-house software. We used a Technics ${ }^{\circledR} \mathrm{SU}$ V-306 M2 amplifier and a Technics ${ }^{\circledR}$ SB-CS6 loudspeaker, and sound pressure was $51-83 \mathrm{~dB}$.

\section{Procedure}

During the entire procedure, participants sat in a comfortable chair in a sound-shielded room. The music stimulus was presented twice. During the first presentation, an EEG was recorded, and subjects were instructed to close their eyes and listen to the music carefully during the whole measurement. The eyes closed condition was chosen to minimize interference from visual input, because it is known that under eyes open condition, baseline arousal is increased) (Barry et al. 2007). Furthermore, subjects were asked not to bite their teeth, and to avoid making movements.

During the second presentation, no EEG was recorded, and subjective arousal level was assessed. Subjects sat at a small table with a computer screen and a mouse. They were asked to rate their subjective arousal level via movements of the mouse. They were instructed to move the mouse forward when they felt a heightened inner tension due to the music, independently of their affective valence. When they felt a reduced inner tension, they moved the mouse backward. The screen turned proportionally red when an increased arousal level was indicated and blue for a reduced arousal level. The rating was recorded and stored for offline analysis with a $100 \mathrm{~Hz}$ sampling rate. Before the measurements, all subjects participated in a 5-min training session to become familiar with the rating instrument. During this training, another piece of music was presented.

After the second presentation, participants completed a questionnaire concerning handedness, age, and gender. Further, musical education (instrument, duration of training, frequency of training, and lessons) and like or dislike of the piece were assessed with a Likert scale, ranging from 1 (maximal dislike) to 10 (maximal like).

\section{EEG Recording and Data Reduction}

During the first presentation, a continuous EEG was recorded using an elastic cap containing $76 \mathrm{Ag}-\mathrm{AgCl}$ electrodes placed according to the international 10-20 system. Two electrodes served as electrooculograms (EOGs) placed below both outer canthi for controlling artifacts caused by eye movements. The average of $\mathrm{C} 3$ and $\mathrm{C} 4$ served as the hardware-defined recording reference. Impedance level was fixed at $<20 \mathrm{k} \Omega$. The EEG was amplified band-pass filtered (10-sec time constant, $120 \mathrm{~Hz}$ low pass), and sampled at $500 \mathrm{~Hz}$ using a Nihon Kohden, Neurofax 1100 system For exact positioning of the onset of the music in the EEG, a marker channel was used. Moreover, the audio track was recorded with the EEG system. 


\section{Main Data Analysis}

The subjective arousal ratings were resampled to a common timescale using spline interpolation as implemented in Matlab by the function "interp1". This timescale had 1-sec intervals, which allowed for a frequency analysis of the EEG while still capturing the dynamics of the ratings. The mean and standard deviation of these interpolated ratings were calculated as individual indices of the mean and variability of arousal level induced by the music.

These indices were correlated with the individual ratings of like or dislike of the music (assessed using a 10-point Likert scale) and the reported hours of practicing music per week using Pearson's correlation coefficient. These correlations were used to identify possible relationships between arousal level during listening and the valence rating after listening, as well as whether music-elicited arousal level habituated or increased with practice.

The mean dynamics of the music-induced fluctuations in arousal were defined as the average, across all subjects, of the 442 interpolated arousal ratings.

The EEG analysis was conducted to identify the spectral correlates of music-induced fluctuations in arousal level, as given by the continuous subjective arousal ratings.

EEG data were analyzed with the Brain Vision Analyzer version 1.05.0005. Eye movements were corrected using independent component analysis (Delorme et al. 2007). Thereafter, channels with excessive artifacts were interpolated (in the average four channels per recording). Further artifacts were removed manually. The data was truncated according to the markers that indicated the onset and offset of the music.

Next, the data were parsed into 442 epochs of 1 -sec duration. All epochs and channels were recomputed to average reference, frequency transformed by means of a fast fourier transform (FFT) and spectral amplitude (defined as square root of spectral power) was computed. For the covariance analysis, for each electrode separately, the mean spectral amplitude across all epochs was removed from each epoch, as we were interested only in the phasic changes of EEG spectral amplitude, and not in the constant tonic part that is represented by the mean. The same was applied to the mean dynamics of arousal level, as we were again only interested in the changes, and not the constant, of arousal rating over time.

The covariance of EEG spectral amplitude with arousal rating was then computed as a weighted sum of the epochs' spectral amplitude, with the average arousal ratings for each epoch serving as weights. This covariance analysis was performed for each subject, excluding epochs with artifacts. Details about this covariance analysis have been given elsewhere (Koenig et al. 2008). While it has been argued that it is advantageous to adjust frequency band borders individually on the basis of their maximum power (Klimesch 1999), we chose to base ours on a recent publication by Jann et al. (2010), which determined its EEG frequency band borders through correlations with fMRI BOLD signals of resting states (Delta, 1-3.5 Hz; Theta1, 3.5-6.25 Hz; Theta2, 6.25-8.2 Hz; Alpha1, 8.2-10.5 Hz; Alpha2, 10.5-14 Hz; Beta1, 14-18.75 Hz; Beta2, 18.75-21.88 Hz; and Beta3, 21.88-30 Hz). In order to test if these covariance maps had common features across subjects, topographic consistency tests were performed for each frequency band (Koenig and Melie-Garcia 2010). This procedure tested the stability of a mean map across subjects by comparing its spatial variance (variance of the values across all electrodes) with the spatial variances of maps that have been obtained from averaging individual maps where the measurements were randomly shuffled across electrodes (randomized mean maps). The shuffling eliminates any consistency of spatial distribution among the individual maps. If the spatial variance of the initiallymeasured mean map is significantly larger than that of most (usually $95 \%$ ) of the randomized mean maps, the test indicates that it is unlikely that the measured mean map has been obtained by averaging individual maps that have nothing in common. If this global test was significant ( $p<0.05$ at 5,000 randomization runs), t-maps (across subjects and against zero) of the covariance maps were computed and displayed.

In previous studies, arousal was found to be confounded by loudness (Krumhansl 1995; Bigand et al. 2006; Granot and Eitian 2011). Therefore, we computed the sound pressure level (SPL) of the music as a function of time using a Hilbert-transformation as implemented in Matlab. We then computed momentary mean arousal values corrected for SPL; this correction was done by subtracting the portion of arousal that could be explained by a linear effect of sound pressure. In the frequency bands where a significant correlation of EEG with arousal was found, we recomputed the covariance maps using the corrected (loudness-independent) arousal values, and tested the resulting covariance maps again for significance.

\section{Results}

Participants' Profile and Subjective Arousal Ratings

In general, there was a good correlation between the trace of the individual arousal ratings across time and the mean arousal rating across time, as indicated by the mean correlation coefficient, $r=0.68$. Sound pressure and mean arousal had a good correlation, with $r=0.62$. However the communality of the ratings persisted when the ratings were corrected for loudness, where a mean correlation 
coefficient of 0.60 was found. This indicated a good amount of communality of the ratings across subjects. There were nevertheless also considerable inter-individual differences, with a range from $r=0.87$ to $r=0.04$ ( $r=$ 0.82 to $r=-0.12$ after loudness correction). The mean explained variance $\left(r^{2}\right)$ was $54.9 \%,(0.43 \%$ after correction) with a range from $76.2 \%(0.68 \%)$ to $1 \%(1 \%)$. Figure 1 shows the mean and individual ratings over time. In the above analyses, significance at a threshold of $p<$ 0.05 ( $\mathrm{df}=440)$ was reached for correlation coefficients above 0.0935 , for a threshold of $p<0.01$, the critical $r$ was 0.123 .

Furthermore, there was a positive correlation between the like or dislike of the music and the mean height of the arousal ratings $\left(r=0.56, r^{2}=0.31, p=0.0194\right.$, df $=17$ ) and the mean height of the arousal ratings and hours practiced per week $\left(r=0.74, r^{2}=0.55, p<0.001\right.$, $\mathrm{df}=17)$.

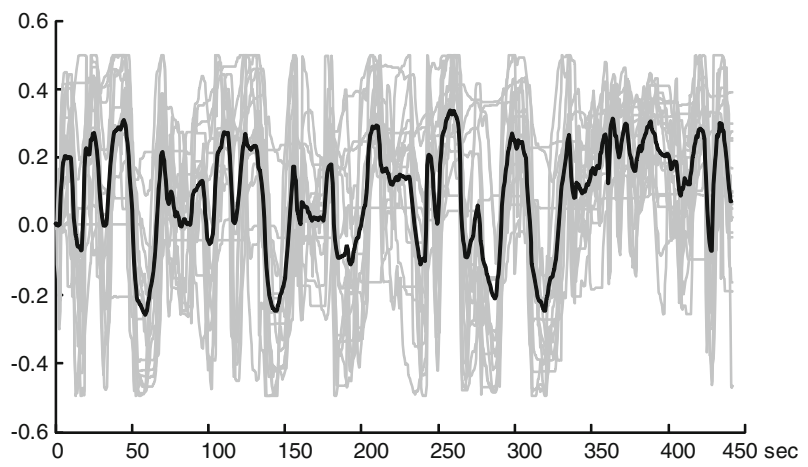

Fig. 1 Subjective arousal ratings (vertical axis, maximal arousal: 0.5, minimal arousal: -0.5 ) as function of time (horizontal axis) during music listening. The mean rating is in black, individual ratings are in grey (Color figure online)

\section{EEG Results}

The topographic consistency test showed significant results for the Theta 2 band $(p=0.02)$, the Alpha1 band $(p=0.02)$, and the Beta 2 band $(p=0.02)$.

The computed covariance maps showed a bilateral parietotemporal suppression of high theta-band activity, a right frontal suppression of lower alpha-band activity, a left parietotemporal suppression of lower alpha-band activity, and a left temporal suppression of beta-band activity during states of high arousal. Since there is literature associating frontal alpha asymmetry with the processing of emotions (Davidson 1995) we compared alpha covariance at F3 with that of $\mathrm{F} 4$, using a paired $t$ test. This $t$ test indicated a significant asymmetry $(t=2.28, \mathrm{df}=18, p=0.035)$, with the left frontal side showing less alpha activity than the right frontal side. When the loudness-corrected arousal was used as a regressor, only the Alpha1 band covariance map remained significant.

Figure 2 shows the covariance maps computed with the average arousal ratings. When covariance maps were computed on the basis of the individual ratings, comparable results were obtained.

A correlation analysis of the association between the covariance maps and the Likert scale ratings yielded no significant results ( $p>0.1$ for all frequency bands).

\section{Discussion}

As indicated by the mean correlation coefficient of 0.68 , there was a considerable consistency between the individual ratings over the entire piece of the symphony, possibly due to the arousal modulation effects of loudness, melody, rhythm, and timbre (Hailstone et al. 2009). In consistence with Schubert (2004) and Ilie and Thompson (2006) in the current
Fig. 2 t-Maps of the individual covariances for all frequency bands (without correction for SPL). Red indicates a power increase with arousal, blue indicates a decrease. Colors above the first color step $( \pm 2.1)$ correspond to $p<0.05$, uncorrected (Color figure online)
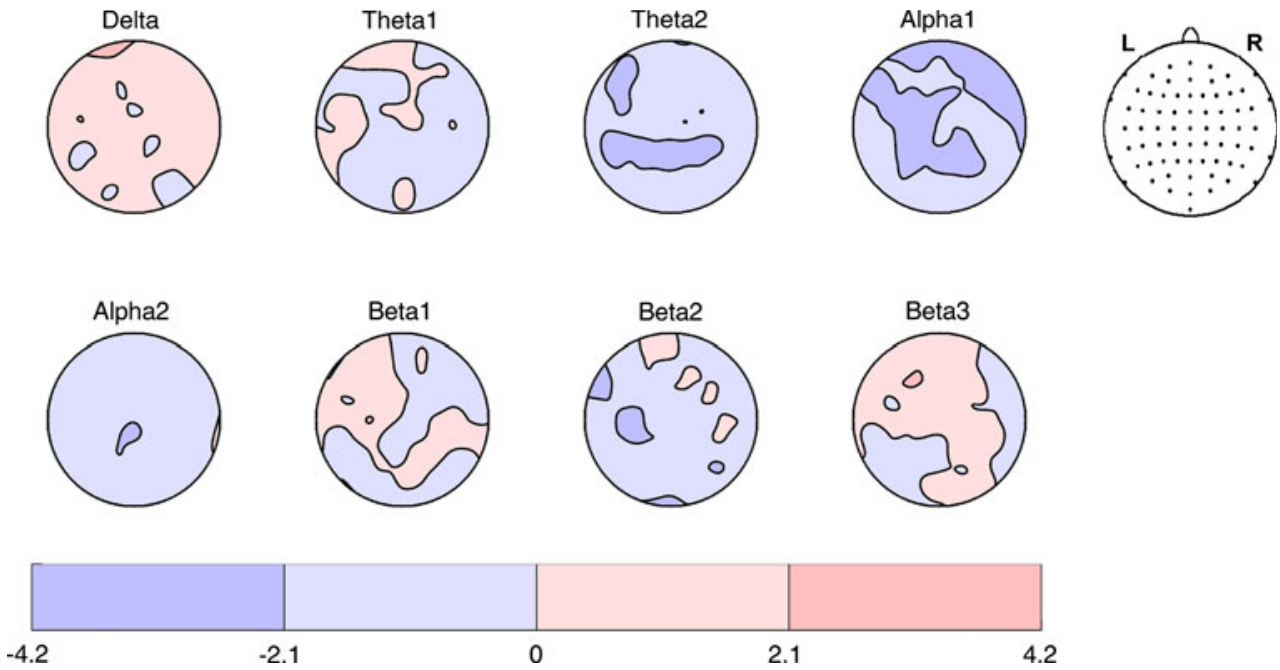
study loudness seems to be the most influencing factor. The interindividual differences seem to suggest that other mechanisms influence musical induced arousal. The positive correlation of mean arousal level and hours practiced furthermore may suggest that during musical training, music specific expectations are continuously reinforced, increasing the subject's responsiveness (James et al. 2008). Several studies showed that arousal levels were modulated according to individual expectations (Krumhansl 1997, 2003; Blood et al. 1999; Koelsch et al. 2008; Salimpoor et al. 2009). The consistency among the ratings may thus be explained with a process that is assumed to be independent of intra-opus and extra-opus knowledge and depends on the realization of expectations (Narmour 1990; Krumhansl 1997).

The correlations between the mean height of ratings and like or dislike of the piece/hours of music practiced per week seems indicative. The elevated ability to segregate concurrent sounds may enable skilled listeners to experience more detail in harmonic progression and thus have more precise expectations (Lee et al. 2009; Zendel and Alain 2009). This may be caused by a heightened ability of segregating concurrent sounds based on harmonicity, which is a very important feature in listening to orchestral music (Lee et al. 2009).

On the other hand, the considerable individual differences of the correlation may reflect "top-down" factors, which may lead to an individual strengthening or weakening of arousal in some passages of the music due to individual learning (James et al. 2008). Further factors may be stable dispositions or based on current mood, which were not quantified in the current study. Other factors mentioned by Scherer (1995), such as the performer's features and the music's structural and contextual features, were constant in this study and thus cannot account for the differences.

The present study showed a bilateral parietotemporal suppression of the upper theta-band activity during periods of high arousal. This finding vanished when the arousal rating was corrected for loudness. This does not mean that the theta changes were independent of arousal, but that they seem to represent those aspects of arousal that strongly covary with loudness. Increased theta activity is often associated with drowsiness (Klimesch 1999), but occurs also during working memory encoding (Klimesch et al. 2006; Krause 2006). Independent of the loudness confounder, our result was contrary to other studies (e.g., Aftanas et al. 2002). A possible explanation is that these studies used aversive stimuli from the IASP to evoke arousal, which was very different from our music stimulus. Furthermore, the diverging findings may result from basic differences in the experiments setup and analysis strategy; their study investigated event-related synchronization and de-synchronization, which quantifies stimulus-induced spectral changes in reference to a prestimulus period, whereas our setup did not include a prestimulus period. The presence or absence of a pre-stimulus period may be relevant, because the recruitment of neuronal resources can increase or decrease spectral power depending on the baseline state of the subject (Pfurtscheller 2006).

Furthermore, we found a right frontal suppression of lower alpha-band activity associated with high arousal ratings. In normally awake subjects, this is classically seen as an index of increased physiological activation (Krause 2006), and has in general been functionally associated with increased attention (Klimesch 1999; Jann et al. 2009). As this was the only activity change independent of loudness, we argue that in our study, these changes were related primarily to the emotional content and not to the dynamics of the music. In this context, frontal alpha-band frequency asymmetry has however been mostly discussed as a moderator and mediator of emotional valence (for a review see Coan and Allan 2004). Nitschke et al. (1999) found in anxiety-associated arousal a specific pattern with right frontal suppression of the alpha-band frequency. It was hypothesized that frontal asymmetry is a correlate of interaction of these cortical structures with subcortical emotion-related areas like the amygdala (Aftanas et al. 2002). Amygdala and orbital frontolateral activation was also shown in response to unexpected chords in a music piece (Koelsch 2009). This increasing tension due to unexpected chords seems to be important in eliciting emotions (Koelsch 2005; James et al. 2008). The importance of limbic structures, namely the amygdala and the parahippocampal cortex, for the understanding of negative emotional content of music was also stressed by lesion studies of Gosselin et al. (2006, 2007). Interestingly, the link among lateralized changes in the alpha band and the simultaneous activation of subcortical regions during the listening of music with different emotional content has recently been established directly by (Flores-Gutierrez et al. 2007), who recorded data with the same stimulus material with EEG and fMRI.

In terms of lateralization, our frontal asymmetry in the alpha band is compatible with the model of Craig (2005) who suggested that right frontal areas are more responsive (show more alpha suppression) to sympathetic (and thus arousing) input than their left counterparts. We also found left parietotemporal suppression of the alpha-band frequency during arousal. Suppression of parietotemporal lower alpha-band activity was previously found during periods of high expectancy (Klimesch et al. 1998).

\section{Conclusion}

Our results indicate that a long piece of music is capable of inducing consistent changes in brain functional states 
associated with arousal and emotions. Those states changes were identified in an analysis across individuals and therefore represent processes that are can be observed independent from eventual individual differences in music preferences. We found a frontal asymmetry in the lower alpha-band, which may be associated with activations of subcortical limbic structures during periods of high arousal in music as shown in other studies.

The results indicate that music is a powerful emotional stimulus. A better understanding may contribute to the development of methods that specifically modify arousal and valence in a therapeutic setting, independent of patients' preferences.

\section{References}

Aftanas LI, Golocheikine SA (2001) Human anterior and frontal midline theta and lower alpha reflect emotionally positive state and internalized attention: high-resolution EEG investigation of meditation. Neurosci Lett 310:57-60

Aftanas L, Varlamov AA, Pavlov SV, Makhnev VP, Reva NV (2002) Time-dependent cortical asymmetries induced by emotional arousal: EEG analysis of event-related synchronization and desynchronization in individually defined frequency bands. Int $\mathrm{J}$ Psychphysiol 44:67-82

Allen JJ, Coan JA, Nazarian M (2004) Issues and assumptions on the road from raw signals to metrics of frontal EEG asymmetry in emotion. Biol Psychol 67:183-218

Barry RJ, Clarke AR, Johnstone SJ, Magee CA, Rushby JA (2007) EEG differences between eyes-closed and eyes-open resting conditions. Clin Neurophysiol 118:2765-2773

Bigand E, Tillmann B, Poulin-Charronnat B (2006) A module for syntactic processing in music? Trends Cogn Sci 10(5):195-196

Blood AJ, Zatorre RJ (2001) Intensely pleasurable responses to music correlate with activity in brain regions implicated in reward and emotion. Proc Natl Acad Sci 20:11818-11823

Blood AJ, Zatorre RJ, Bermudez P, Evans AC (1999) Emotional responses to pleasant and unpleasant music correlate with activity in paralimbic brain regions. Nat Neurosci 2(4):382-387

Coan JA, Allan JJ (2004) Frontal EEG asymmetry as a moderator and mediator of emotion. Biol Psychol 67:7-49

Craig AD (2005) Forebrain emotional asymmetry: a neuroanatomical basis? Trends Cogn Sci 9:566-571

Davidson RJ (1995) Brain asymmetry. MIT Press, Cambridge

Davidson RJ (2004) What does the prefrontal cortex "do" in affect: perspectives on frontal EEG asymmetry research. Biol Psychol 67:219-233

Dellacherie D, Hugueville RL, Peretz I, Samson S (2010) The effect of musical experience on emotional self-reports and psychophysiological responses to dissonance. Psychophysiology 48(3): 337-349

Delorme A, Sejnowski T, Makeig S (2007) Enhanced detection of artifacts in EEG data using higher-order statistics and independent component analysis. NeuroImage 34(4):1443-1449

Eldar E, Ganor O, Admon R, Bleich A, Hendler T (2007) Feeling the real world: limbic response to music depends on related content. Cereb Cortex 17(12):2828-2840

Flores-Gutierrez EO, Diaz JL, Barrios FA, Favila-Humara R, Guevara MA, del Rio-Portilla Y, Corsi-Cabrera M (2007) Metabolic and electric brain patterns during pleasant and unpleasant emotions induced by music masterpieces. Int J Psychophysiol 65:69-84
Frego R (1999) Effects of aural and visual conditions on response to perceived artistic tension in music and dance. J Res Music Edu 47:31-43

Gable PA, Harmon-Jones E (2009) Postauricular reflex responses to pictures varying in valence and arousal. Psychophysiology 46(3):487-490

Gosselin N, Samson S, Adolphs R, Noulhiane M, Roy M, Hasboun D, Baulac M, Peretz I (2006) Emotional responses to unpleasant music correlates with damage to the parahippocampal cortex. Brain J Neurol 129:2585-2592

Gosselin N, Peretz I, Johnsen E, Adolphs R (2007) Amygdala damage impairs emotion recognition from music. Neuropsychologia 45:236-244

Granot R, Eitian Z (2011) Musical tension and the interaction of dynamic auditory parameters. Music Percept 28(3):219-245

Hailstone JC, Omar R, Henley SM, Frost C, Kenward MG, Warren JD (2009) It's not what you play, it's how you play it: timbre affects perception of emotion in music. Q J Exp Psychol 62(11):1-15

Heller W, Nitschke JB, Lindsay DL (1997) Neuropsychological correlates of arousal in self-reported emotion. Cogn Emotion 11(4):383-402

Huron D (2001) Is music an evolutionary adaptation? Ann N Y Acad Sci 930:43-61

Huron D (2006) Sweet anticipation. MIT Press, Cambridge

Ilie G, Thompson W (2006) A comparison of the acoustic cues in music nd speech for three dimensions of affect. Music Percept 23:319-329

James CE, Britz J, Vuilleimier P, Hauert CA, Michel CM (2008) Early neuronal responses in right limbic structures mediate harmony incongruity processing in musical experts. Neuroimage 42(4):1597-1608

Jann K, Dierks T, Boesch C, Kottlow M, Strik W, Koenig T (2009) BOLD correlates of EEG alpha phase-locking and the fMRI default mode network. NeuroImage 45(3):903-916

Jann K, Kottlow M, Dierks T, Boesch C, Koenig T (2010) Topographic electrophysiological signatures of FMRI resting state networks. PLoS ONE 5(9):e12945

Juslin PN, Vastfjall D (2008) Emotional responses to music: the need to consider underlying mechanisms. Behav Brain Sci 31(5): 559-621

Klimesch W (1999) EEG alpha and theta oscillations reflect cognitive and memory performance: a review and analysis. Brain Res Brain Res Rev 29(2-3):169-195

Klimesch W, Doppelmayr M, Russegger H, Pachinger T, Schwaiger J (1998) Induced alpha band power changes in the human EEG and attention. Neurosci Lett 244(2):73-76

Klimesch W, Doppelmayr M, Hanlsmayr S (2006) Upper alpha ERD absolute power: their meaning for memory performance. Prog Brain Res 159:151-165

Koelsch S (2005) Investigating emotion with music: neuroscientific approaches. Ann N Y Acad Sci 1060:412-418

Koelsch S (2009) A neuroscientific perspective on music therapy. Ann N Y Acad Sci 1169:374-384

Koelsch S, Fritz T, v. Cramon DY, Muller K, Friederici AD (2006) Investigating emotion with music: an fMRI study. Hum Brain Mapp 27(3):239-250

Koelsch S, Fritz T, Schlaug G (2008) Amygdala activity can be modulated by unexpected chord functions during music listening. NeuroReport 19(18):1815-1819

Koenig T, Melie-Garcia L (2010) A method to determine the presence of averaged event-related fields using randomization tests. Brain Topogr 23(3):233-242

Koenig T, Melie-Garcia L, Stein M, Strik W, Lehmann C (2008) Establishing correlations of scalp field maps with other experimental variables using covariance analysis and resampling methods. Clin Neurophysiol 119:1262-1270 
Krause CM (2006) Cognition- and memory-related ERD/ERS responses in the auditory stimulus modality. Prog Brain Res 159:197-207

Krumhansl C (1995) Music psychology and music theory: problems and prospects. Music Theory Spectr 17(1):53-80

Krumhansl C (1997) An exploratory study of musical emotions and psychophysiology. Can J Exp Psychol 51(4):336-352

Krumhansl C (2003) Experimental strategies for understanding the role of experience in music cognition. Ann N Y Acad Sci 999: 414-428

Lee KM, Skoe E, Kraus N, Ashley R (2009) Selective subcortical enhancement of musical intervals in musicians. J Neurosci 29(18): $5832-5840$

Madson AT, Silverman MJ (2010) The effect of music therapy on relaxation, anxiety, pain perception, and nausea in adult solid organ transplant patients. J Music Ther 47(3):220-232

Meadows ME, Kaplan RF (1994) Dissociation of autonomic and subjective responses to emotional slides in right hemisphere damaged patients. Neuropsychologia 32:847-856

Menon V, Levitin DJ (2005) The rewards of music listening: response and physiological connectivity of the mesolimbic system. NeuroImage 28(1):175-184

Meyer L (1956) Emotion and meaning in music. University of Chicago Press, Chicago

Moradipanah F, Mohammadi E, Mohammadil AZ (2009) Effect of music on anxiety, stress, and depression levels in patients undergoing coronary angiography. East Mediterr Health J 15(3): 639-647

Muller TJ, Federspiel A, Fallgatter AJ, Strik WK (1999) EEG signs of vigilance fluctuations preceding perceptual flips in multistable illusionary motion. NeuroReport 10(16):3423-3427

Narmour E (1990) The analysis and cognition of basic melodic structures. University of Chicago Press, Chicago

Nitschke JB, Heller W, Palmieri PA, Miller GA (1999) Contrasting patterns of brain activity in anxious apprehension and anxious arousal. Psychophysiology 36:628-637

Ochsner KN, Ray RR, Hughes B, McRae K, Cooper JC, Weber J, Gabrieli JD, Gross JJ (2009) Bottom-up and top-down processes in emotion generation: common and distinct neural mechanisms. Psychol Sci 20:1322-1331

Panksepp J, Bernatzky G (2002) Emotional sounds and the brain: the neuro-affective foundations of musical appreciation. Behav Proc 60:133-155

Patel AD (2003) Language, music, syntax and the brain. Nat Neurosci 6(7):674-681
Pfurtscheller G (2006) The cortical activation model (CAM). Prog Brain Res 159:19-27

Russel J (1980) A circumplex model of affect. J Pers Soc Psychol 39(6):1161-1178

Salimpoor VN, Benovoy M, Longo G, Cooperstock JR, Zatorre RJ (2009) The rewarding aspects of music listening are related to degree of emotional arousal. PLoS ONE 4(10):e7487

Sammler D, Grigutsch M, Fritz T, Koelsch S (2007) Music and emotion: electrophysiological correlates of the processing of pleasant and unpleasant music. Psychophysiology 44(2):293-304

Sarter M, Givens B, Bruno JP (2001) The cognitive neuroscience of sustained attention: where top-down meets bottom-up. Brain Res Brain Res Rev 35:146-160

Scherer KR (1995) Expression of emotion in voice and music. J Voice 9(3):235-248

Schubert E (2004) Modeling perceived emotion with continuous musical features. Music Percept 21:561-585

Steinbeis N, Koelsch S, Sloboda JA (2006) The role of harmonic expectancy violations in musical emotions: evidence from subjective, physiological, and neural responses. J Cogn Neurosci 18:1380-1393

Suetsugi M, Mizuki Y, Ushijima I, Kobayashi T, Tsuchiya K, Aoki T (2000) Appearance of frontal midline theta activity in patients with generalized anxiety disorder. Neuropsychobiology 41:108-112

Tsang CD, Trainor LJ, Santesso DL, Tasker SL, Schmidt L, Aalto S (2001) Frontal EEG responses as a function of affective musical features. Ann N Y Acad Sci 930:439-442

Tsuno N, Shigeta M, Hyoki K, Faber PL, Lehmann D (2004) Fluctuations of source locations of EEG activity during transition from alertness to sleep in Alzheimer's disease and vascular dementia. Neuropsychobiology 50(3):267-272

Wu D, Li C, Yin Y, Zhou C, Yao D (2010). Music composition from the brain signal: representing the mental state by music. Comput Intell Neurosci. doi:10.1155/2010/267671

Zare M, Ebrahimi AA, Birashk B (2010) The effects of music therapy on reducing agitation in patients with Alzheimer's disease, a prepost study. Int J Geriatr Psychiatry 25(12):1309-1310

Zendel BR, Alain C (2009) Concurrent sound segregation is enhanced in musicians. J Cogn Neurosci 21(8):1488-1498

Zentner M, Grandjean D, Scherer KR (2008) Emotions evoked by the sound of music: characterization, classification, and measurement. Emotion 8(4):494-521

Zhang J, Zhou R, Oei T (2011) The effects of valence and arousal on hemispheric asymmetry of emotion: evidence from event-related potentials. J Psychophysiol 25:95-103 